\title{
Calibration of Instrument Current Transformer Test Sets
}

\section{Karel Draxler}

Dept. of Measurement Faculty of El. Engineering Czech Technical University

Prague, Czech Republic draxler@fel.cvut.cz

\author{
Jan Hlaváček
}

Dept. of El. Power Engineering

Faculty of El. Engineering

Czech Technical University

Prague, Czech Republic

jan.hlavacek@fel.cvut.cz
Renata Styblíková

Dept. of Electromagnetic

Measurements

Czech Metrology Institute

Prague, Czech Republic

rstyblikova@cmi.cz

\begin{abstract}
The paper presents the basic layout for instrument current transformer calibration using comparative method with a standard. The error difference is evaluated using electronic transformer test sets. Calibrating procedure of these systems using error simulation is also described. In conclusion, results obtained using described procedure are presented.
\end{abstract}

Keywords-instrument current transformer, calibration, ratio error, phase displacement

\section{INTRODUCTION}

Calibration of instrument current transformers (ICT) [1], [2], [3], [4], [5] at $50 \mathrm{~Hz}$ frequency is usually performed using a comparative method at layout according to Fig. 1.

Primary windings of the two transformers are connected in series and supplied by a common current $I_{1}$ from a current source. The burden of the ICT under test is connected in series with the evaluation circuit.

Secondary currents of a standard $I_{2 \mathrm{~N}}$ and an ICT under test $I_{2 \mathrm{X}}$ are led to a system where the ratio error is evaluated as a deviation of the magnitude and the phase displacement as the phase shift of phasors $I_{2 \mathrm{X}}-I_{2 \mathrm{~N}}$ as shown in the phasor diagram in Fig. 2. The error difference may be expressed in the form

$$
\varepsilon_{\mathrm{ID}}=\varepsilon_{\mathrm{IX}}-\varepsilon_{\mathrm{IN}}=\frac{I_{\mathrm{X}}-I_{\mathrm{N}}}{I_{\mathrm{N}}} 100=\frac{\Delta I_{\mathrm{Re}}}{I_{\mathrm{N}}} 100
$$

$$
\delta_{\mathrm{ID}}=\delta_{\mathrm{IX}}-\delta_{\mathrm{IN}}=\operatorname{tg} \delta_{\mathrm{ID}}=\frac{\Delta I_{\mathrm{Im}}}{I_{\mathrm{N}}-\Delta I_{\mathrm{Re}}},
$$

where $\varepsilon_{\mathrm{IX}}$ and $\varepsilon_{\mathrm{IN}}$ are ratio errors of an ICT under test and a standard (\%), $\delta_{\mathrm{IX}}$ and $\delta_{\mathrm{IN}}$ are phase displacements of an ICT under test and a standard (rad), $I_{2 \mathrm{X}}$ and $I_{2 \mathrm{~N}}$ are magnitudes of secondary current of an ICT under test and a standard (A), $\Delta I_{\text {Re }}$ and $\Delta I_{\text {Im }}$

Figure 2. Phasor diagram of secondary currents

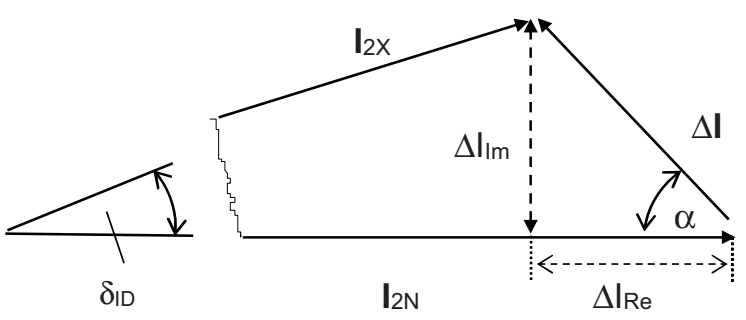

Figure 1. Basic layout for ICT calibration using comparative method

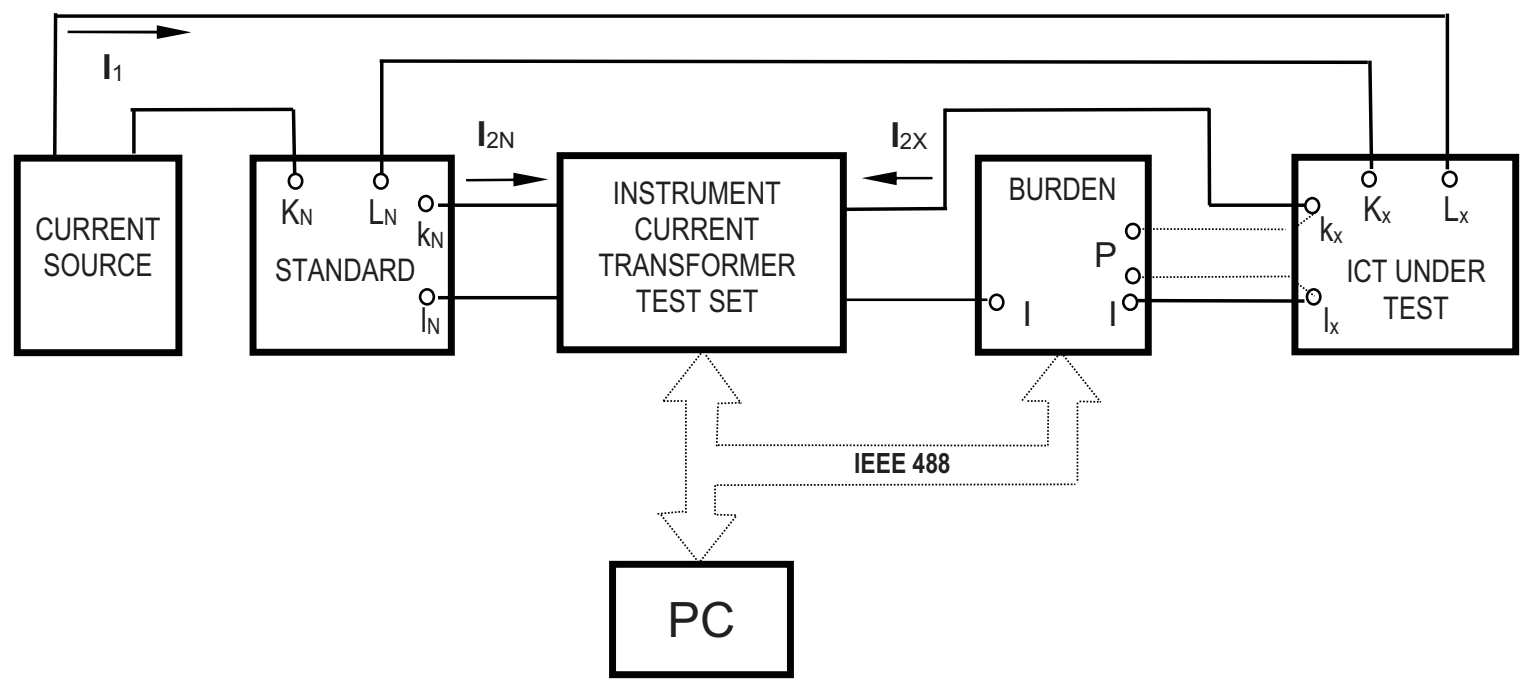


are magnitudes of rectangular components of the phasor of the differential current $\Delta I$ regarding to the current $I_{\mathrm{N}}(\mathrm{A})$.

Eq. (1) and (2) or their simplifications are valid only at the assumption that errors of a standard are very small (eg. $\varepsilon_{\mathrm{IN}} \leq 0.01 \%$ ) and when

$$
\Delta I \ll I_{N}, \quad \Delta \mathrm{I}_{\mathrm{Re}} \ll \mathrm{I}_{\mathrm{N}}, \Delta \mathrm{I}_{\mathrm{Im}} \ll \mathrm{I}_{\mathrm{N}} .
$$

Resulting errors of the ICT under test are given as

$$
\varepsilon_{\mathrm{IX}}=\varepsilon_{\mathrm{ID}}+\varepsilon_{\mathrm{IN}} \quad \delta_{\mathrm{IX}}=\delta_{\mathrm{ID}}+\delta_{\mathrm{IN}}
$$

where $\varepsilon_{\mathrm{ID}}$ and $\delta_{\mathrm{ID}}$ measured by transformer test set.

Mostly electronic systems are currently used for error difference $\varepsilon_{\mathrm{ID}}$ and $\delta_{\mathrm{ID}}$ evaluation between a standard and an ICT under test. Older systems evaluate the difference from a voltage drop across a resistor so that only transformers with identical transformation ratios can be compared. Newer systems [6], [7] use a differential current transformer at their input, allowing comparisons of transformers with different ratios in certain range of their primary and secondary currents. The block diagram of an ICT test set with input differential current transformer is shown in Fig. 3.

Secondary circuits of the ICT under test "X" loaded by a burden ad $B$ and of the standard " $\mathrm{N}$ " are connected to the primary winding of the differential current transformer DT. The impedance of the system on the $\mathrm{k}_{\mathrm{X}}-\mathrm{l}_{\mathrm{X}}$ and $\mathrm{k}_{\mathrm{N}}-\mathrm{l}_{\mathrm{N}}$ terminals does not exceed 30 $\mathrm{m} \Omega$, so the system only minimally increases burden of the two transformers. The ratio of the secondary currents and the number of turns of the windings DT must satisfy the condition $\mathrm{N}_{\mathrm{dX}} / \mathrm{N}_{\mathrm{dN}}=\mathrm{I}_{2 \mathrm{~N}} / \mathrm{I}_{2 \mathrm{X}}$. Choosing a DT number ratio allows comparing ICT with different transformation ratios and different secondary currents. The reference voltage $\boldsymbol{U}_{\mathrm{N}}$ and the error voltage $\Delta \boldsymbol{U}$ are sampled and led to a microprocessor where error difference components are calculated, which can be expressed as

$$
\begin{gathered}
\varepsilon_{\mathrm{ID}}=\frac{N_{\mathrm{S}} \Delta I_{\mathrm{Re}}}{N_{\mathrm{dN}} I_{2 \mathrm{~N}}}=\frac{N_{\mathrm{S}} \Delta U_{\mathrm{Re}}}{R N_{\mathrm{dN}} I_{2 \mathrm{~N}}} \\
\delta_{\mathrm{ID}}=\operatorname{tg} \delta_{\mathrm{ID}}=\frac{N_{\mathrm{S}} \Delta U_{\mathrm{Im}}}{R\left(N_{\mathrm{dN}} I_{2 \mathrm{~N}}-N_{\mathrm{S}} \Delta I_{\mathrm{Re}}\right)}
\end{gathered}
$$

where $N_{\mathrm{S}}$ is number of DT secondary windings, $N_{\mathrm{S}}$ $\Delta \boldsymbol{I}_{\mathrm{Re}}$ and $N_{\mathrm{S}} \Delta \boldsymbol{I}_{\mathrm{Im}}$ is the real and imaginary part of the phasor $N_{\mathrm{S}} \Delta \boldsymbol{I}$ regarding to the phasor $N_{\mathrm{dN}} \boldsymbol{I}_{2 \mathrm{~N}}$. The resistor $R$ is in the feedback of the current/voltage converter $\mathrm{I} / \mathrm{U}$ is connected in DT secondary. A typical example of a system working on this principle is the Tettex 2767 system [6]. From Eq. (4) it is obvious that
Figure 3. Layout of a system for ICT error evaluation with different ratios

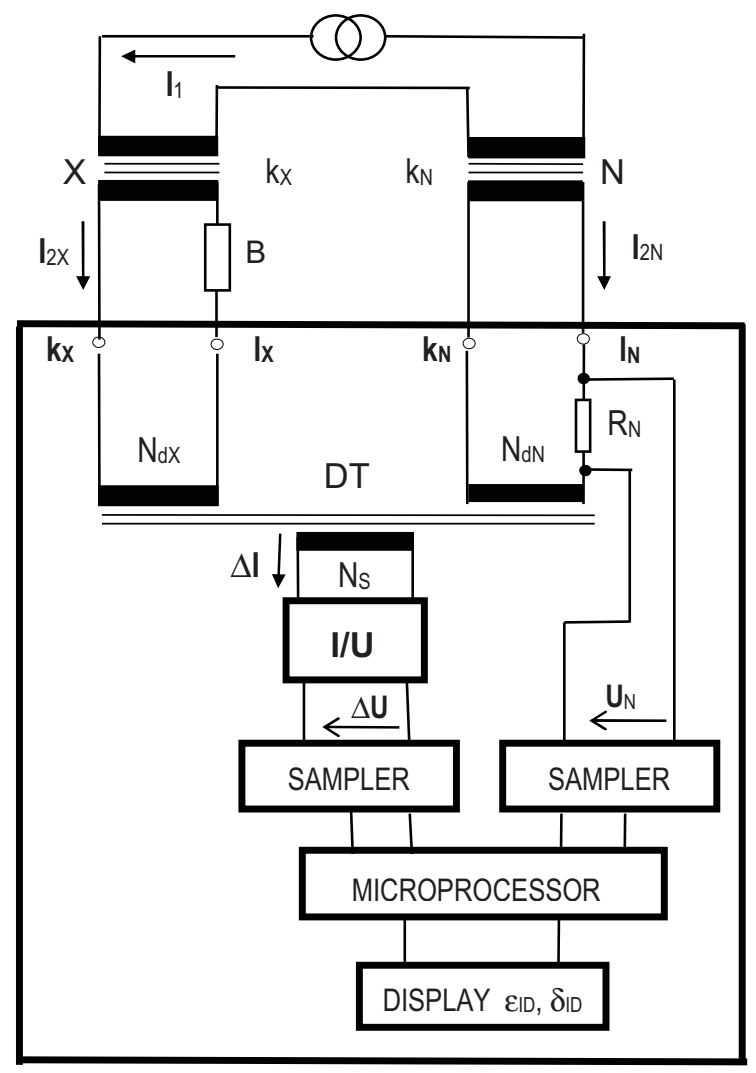

the measurement accuracy of the difference $\varepsilon_{\mathrm{ID}}$ and $\delta_{\mathrm{ID}}$ influence the resulting accuracy of error measurement $\varepsilon_{\mathrm{ID}}$ and $\delta_{\mathrm{ID}}$. So the calibration of the transformer test set described in the following text is an important part by ICT error measurement.

\section{CALIBRATION OF THE SYSTEM}

The layout in Fig. 4 shows arrangement of a system for instrument current transformer test sets calibration by error simulation using an additional current source. Terminals $\mathrm{k}_{\mathrm{X}}$ and $\mathrm{l}_{\mathrm{N}}$ of the test set are serially connected and the circuit for evaluating the secondary currents $\boldsymbol{I}_{2 \mathrm{X}}$ and $\boldsymbol{I}_{2 \mathrm{~N}}$ is fed from a current source by a common current $\boldsymbol{I}_{2}$. If $\Delta I_{2}=0$ and $I_{2 \mathrm{X}}=$ $I_{2 \mathrm{~N}}=I_{2}$, for values of the ratio error $\varepsilon_{\mathrm{IX}}$ and phase displacement $\delta_{\mathrm{IX}}$ equal zero in an ideal case. Additional errors are simulated using a current source $\Delta \boldsymbol{I}_{2}$ with adjustable magnitude and phase shift regarding to the $\Delta \boldsymbol{I}_{2}$. When the phasor $\Delta \boldsymbol{I}_{2}$ is in phase with the phasor $\boldsymbol{I}_{2}$ then according to Fig. $2, \Delta \boldsymbol{I}_{2}=\Delta \boldsymbol{I}_{\mathrm{Re}}$ and only the ratio error is simulated which may be

Figure 4. Error simulation by an additional current source

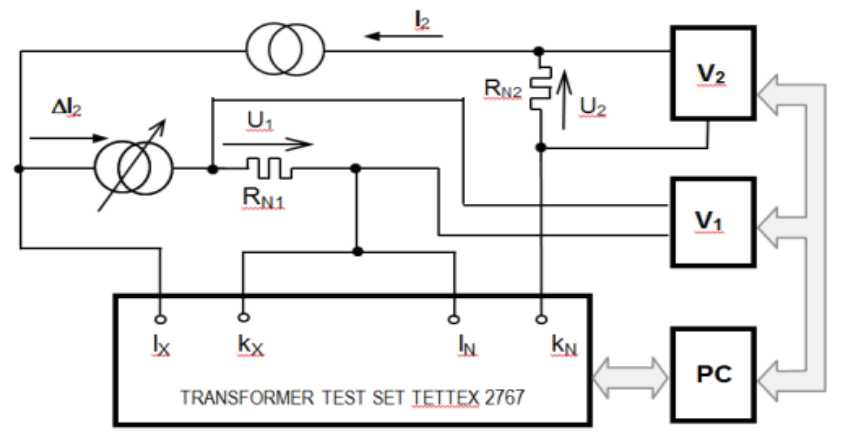


expressed as

$$
\varepsilon_{\mathrm{I}}=\frac{\Delta I_{2 \varepsilon}}{I_{2 \varepsilon}} 100=\frac{U_{1 \varepsilon}}{U_{2 \varepsilon}} \frac{R_{\mathrm{N} 2}}{R_{\mathrm{N} 1}} 100
$$

where $\varepsilon_{\mathrm{I}}$ is simulated ratio error (\%), $U_{1 \varepsilon}$ and $U_{2 \varepsilon}$ are voltage drops across standard resistors $R_{\mathrm{N} 1}$ and $R_{\mathrm{N} 2}$ by ratio error simulation $\varepsilon_{\mathrm{I}}(\mathrm{V})$.

$$
\delta_{I}=\operatorname{tg} \delta_{I}=\frac{\Delta I_{2 \delta}}{I_{2 \delta}}=\frac{U_{1 \delta}}{U_{2 \delta}} \frac{R_{\mathrm{N} 2}}{R_{\mathrm{N} 1}}
$$

where $\delta_{\mathrm{I}}$ is simulated phase displacement ( $\left.\mathrm{rad}\right), U_{1 \delta}$ and $U_{2 \delta}$ are voltage drops across standard resistors $R_{\mathrm{N} 1}$ and $R_{\mathrm{N} 2}$ by phase displacement simulation $\delta_{\mathrm{I}}(\mathrm{V})$.
The calibration is performed according to the following procedure:

i) Setting the rated value of the secondary current $\left(\right.$ eg. $\left.I_{2}=5 \mathrm{~A}\right)$

ii) Setting an approximate value of the error current $\Delta I_{2}$

iii) Setting the phase shift so way that the phase displacement $\delta_{\mathrm{I}}=0$

iv) Setting the required value of ratio error $\varepsilon_{\mathrm{I}}$

v) Reading of simulated error and individual voltages using a PC controlled system

vi) Calculation of true simulated values according to (7) and (8).

The procedure is analogous for phase displacement simulation.

The standard measurement uncertainty [8] of the correct value of the simulated ratio error given by (7) may be generally expressed as

$\mathrm{u}\left(\varepsilon_{\mathrm{I}}\right)=\sqrt{\left(\frac{\partial \mathrm{u}\left(\varepsilon_{\mathrm{I}}\right)}{\partial U_{1}} \mathrm{u}\left(U_{1}\right)\right)^{2}+\left(\frac{\partial \mathrm{u}\left(\varepsilon_{\mathrm{I}}\right)}{\partial R_{\mathrm{N} 2}} \mathrm{u}\left(R_{\mathrm{N} 2}\right)\right)^{2}\left(\frac{\partial \mathrm{u}\left(\varepsilon_{\mathrm{I}}\right)}{\partial U_{2}} u\left(U_{2}\right)\right)^{2}\left(\frac{\partial \mathrm{u}\left(\varepsilon_{\mathrm{I}}\right)}{\partial R_{\mathrm{N} 1}} \mathrm{u}\left(R_{\mathrm{N} 1}\right)\right)^{2}}$,

where $\mathrm{u}\left(U_{1}\right), \mathrm{u}\left(U_{2}\right), \mathrm{u}\left(R_{\mathrm{N} 1}\right)$ and $\mathrm{u}\left(R_{\mathrm{N} 2}\right)$ are uncertainties of measured voltages and sensing resistors.

Expressing individual partial derivatives the standard uncertainty is given as

$\mathrm{u}\left(\varepsilon_{\mathrm{I}}\right)=\sqrt{\left(\frac{R_{\mathrm{N} 2}}{U_{2} R_{\mathrm{N} 1}} \mathrm{u}\left(U_{1}\right)\right)^{2}+\left(\frac{U_{1}}{U_{2} R_{\mathrm{N} 1}} \mathrm{u}\left(R_{\mathrm{N} 2}\right)\right)^{2}+\left(-\frac{U_{1} R_{\mathrm{N} 2}}{U_{2}^{2} R_{\mathrm{N} 1}} \mathrm{u}\left(U_{2}\right)\right)^{2}+\left(-\frac{U_{1} R_{\mathrm{N} 2}}{U_{2} R_{\mathrm{N} 1}^{2}} \mathrm{u}\left(R_{\mathrm{N} 1}\right)\right)}$.

Uncertainties for phase displacements simulated are expressed analogously.

\section{AUXILIARY ELECTRONIC CIRCUITS}

Current sources of the calibration system can be implemented either as a voltage source with a serial resistor or as a voltage-current converter with an operational amplifier. Use of the first variant can be applied to the source of error current $\Delta \boldsymbol{I}_{2}$. The nominal value of the secondary current $I_{2}$ is mostly $5 \mathrm{~A}$ or $1 \mathrm{~A}$, respectively. The simulated ratio error $\varepsilon_{\mathrm{I}}=5 \%$ corresponds to the error current $\Delta I=0.25 \mathrm{~A}$; in case of phase displacement, the situation is analogous. In this case, the current source can be realized as a $75 \mathrm{~V}$ voltage source with a $300 \Omega$ resistor in series. The voltage source must be provided with a transformer at the output to provide a zero DC component of the current $I_{2}$. Since the impedance at $\mathrm{k}_{\mathrm{X}}-\mathrm{l}_{\mathrm{X}}$ and $\mathrm{k}_{\mathrm{N}}-\mathrm{l}_{\mathrm{N}}$ terminals does not exceed $30 \mathrm{~m} \Omega$ and the current source of $I_{2}$ is implemented using an operational amplifier, the design of the source of the error current $\Delta I_{2}$ is satisfactory. A block diagram of the arrangement of this source is shown in Fig. 5.
The basic element for the realization of the continuous phase shift is a resolver [9]. If the voltages applied to the stator windings have the $90^{\circ}$ phase shift, the individual voltages can be expressed as

$$
\begin{aligned}
& \boldsymbol{U}_{1 \mathrm{~S}}=U_{1} \cos \omega \mathrm{t}, \\
& \boldsymbol{U}_{2 \mathrm{~S}}=U_{1} \sin \omega \mathrm{t}, \\
& \boldsymbol{U}_{\mathrm{R}}=k_{\mathrm{R}} U_{1} \cos (\omega \mathrm{t}+\varphi),
\end{aligned}
$$

where $\boldsymbol{U}_{1 \mathrm{~S}}$ and $\boldsymbol{U}_{2 \mathrm{~S}}$ are supply voltages of the stator windings $(\mathrm{V}), \boldsymbol{U}_{\mathrm{R}}$ is the voltage at the rotor winding output, $k_{\mathrm{R}}$ is the transformation factor of the resolver, and $\varphi$ is angular rotation of the rotor relative to the base position.

The phase shift $90^{\circ}$ of the voltage for supplying the stator windings is obtained by means of an integrator with the cutoff frequency substantially lower than 50 $\mathrm{Hz}$. The output voltage from the rotor of the resolver is adjusted by a high input resistance separating amplifier and led to a comparator to obtain a rectangular waveform with stable amplitude given by the reference voltage. From it, a triangular waveform

Figure 5. Block diagram of analog phase shift voltage source

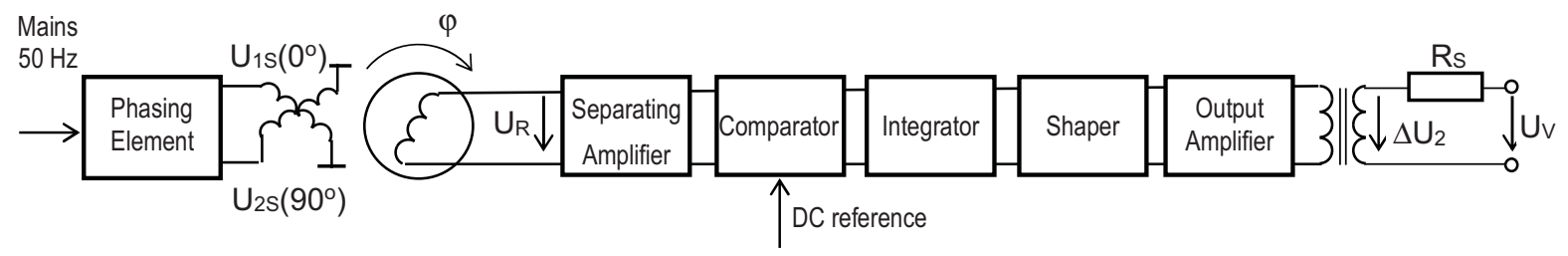


is created by means of an integrator, then led to a special shaper, where a sinusoidal waveform is obtained. The phase shift of the $\boldsymbol{U}_{\mathrm{V}}$ voltage corresponds to the angle of rotation of the resolver rotor. The signal processing behind the rotor of the resolver ensures stable amplitude and constant minimum distortion of the output voltage $\Delta U_{2}$. The value of the serial resistor $R_{\mathrm{S}}$ is switched according to the range of the error current $\Delta I_{2}$.

The circuit diagram of the secondary current source $I_{2}$ is shown in the Fig. 6 . The basis is a voltageto-current converter implemented by the operational amplifier LM 3875. The output circuit is separated by a current transformer CT with a $1 \mathrm{~A} / 10 \mathrm{~A}$ ratio, which separates the direct current component of the $I_{1}$. If the burden $R_{\mathrm{Z}}$ in the secondary circuit does not exceed

\section{CONCLUSION}

The described arrangement is used in the CMI, Laboratory of primary metrology to calibrate systems for the CT error evaluation with comparison to the standard. These are Tettex 2763, Tettex 2761, Tettex 2767 [6] and also Zera WM 1000I, WM 3000I [7], and Oltest CA507. The calibration process is controlled by a PC, allowing immediate processing of the results. The PC program allows immediately checking the accuracy of the instrument current transformer test set given by manufacturer. The methodic error of measurement can be caused if the proper current sources $I_{2}$ and $\Delta I_{2}$ are not used. Stability of the simulated errors is ensured by common phase lock of the current source $I_{2}$ and the source of the error current $\Delta \mathrm{I}_{2}$ on the $50 \mathrm{~Hz}$ network.

Figure 6 . The circuit diagram of the secondary current source $I_{2}$

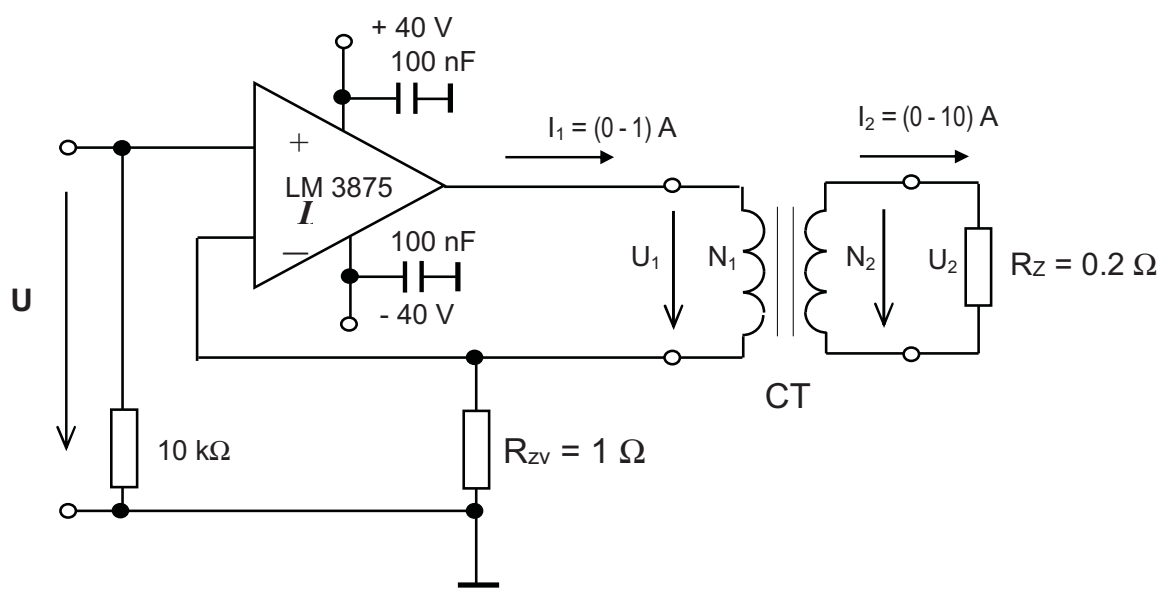

$0.2 \Omega$, then this connection is satisfactory for the calibration of the instrument current transformer test set.

\section{ACCURACY OF CALIBRATION}

At the secondary current of $5 \mathrm{~A}$ according to Fig. 4, the current ratio error simulation $\varepsilon_{\mathrm{I}}=0.005 \%$ is corresponding to the error current $\Delta I_{2}=0.25 \mathrm{~mA}$, so when using a sensing standard resistor $R_{\mathrm{N} 1}=10 \mathrm{k} \Omega \pm 0.05 \%$ then the measured voltage $U_{1}=2.5 \mathrm{~V}$. To measure the current $I_{2}$ the standard resistor $R_{\mathrm{N} 2}=0.1 \Omega \pm 0.05 \%$ is used, so the $U_{2}=0.5 \mathrm{~V}$. When HP $34401 \mathrm{~A}$ voltmeters are used in the voltage measurement system, the substitution (10) and the extension coefficient $k=2$ will give the true value of simulated current ratio error $\varepsilon_{\mathrm{I}}= \pm(0.005 \pm 0.000016) \%$. Similarly, when simulating a phase displacement error of $\delta_{\mathrm{I}}=50 \mu \mathrm{rad}$ $=0.172$ angular minutes. While maintaining the uncertainty of the sensing resistors $R_{\mathrm{N} 1}$ and $R_{\mathrm{N} 2}$ and the uncertainties of measured voltages, with the extension coefficient $k=2$, the true value of the simulated phase displacement is then $\delta_{\mathrm{I}}=(0.172 \pm 0.0005)$ angular minutes. Similarly, it can be shown that even for other simulated error values, the true value uncertainty is significantly less than the measured value uncertainty given by the manufacturer.

\section{REFERENCES}

[1] Instrument transformers - Part 2: Additional requirements for current transformers, Doc. IEC 61869-2:2012, 2012.

[2] S. Siegenthaler and C. Mester, "A Computer-Controlled Calibrator for Instrument Transformer Test Sets," in IEEE Transactions on Instrumentation and Measurement, vol. 66, no. 6, pp. 1184-1190, June 2017.

[3] E. Mohns, G. Roeissle, S. Fricke and F. Pauling, "An AC Current Transformer Standard Measuring System for Power Frequencies," in IEEE Transactions on Instrumentation and Measurement, vol. 66, no. 6, pp. 1433-1440, June 2017.

[4] G. Rietveld, L. Jol, H. E. van den Brom and E. So, "HighCurrent CT Calibration Using a Sampling Current Ratio Bridge," in IEEE Transactions on Instrumentation and Measurement, vol. 62, no. 6, pp. 1693-1698, June 2013.

[5] J. K. Jung ; E. So ; Y. T. Park ; M.Kim, "KRISS-NRC Intercomparisons of Calibration Systems for Instrument Transformers with Many Different Ratios at Power Frequency", in IEEE Transactions on Instrumentation and Measurement, vol. 58 , Issue: 4, pp. 1023-1028 Year 2009.

[6] Tettex Instruments, Automatic instrument transformer test set. [Online]. Available: http://www.tettex.com

[7] WM 1000I, Current transformer measuring bridge, [Online]. Available: https//www.zera.de

[8] BIPM, Guide to the Expression of Uncertainty in Measurement, 2008

[9] M. Benammar, A. S. P. Gonzales, "A Novel PLL Resolver Angle Position Indicator," in IEEE Transactions on Instrumentation and Measurement, vol. 65, no. 1, pp. 123131, Jan. 2016. 